\title{
Estrategias de búsqueda visual en conductores expertos y noveles durante la visualización de escenas de tráfico
}

\author{
Juan M. Gómez-Valadés ${ }^{1}$, Vicente Luis ${ }^{1 *}$, Raúl Reina², Rafael Sabido² y Francisco J. Moreno² \\ ${ }^{1}$ Laboratorio de Aprendizaje y Control Motor, Facultad de Ciencias del Deporte, Universidad de Extremadura, España \\ ${ }^{2}$ Centro de Investigación del Deporte, Universidad Miguel Hernández de Elche, España
}

\begin{abstract}
Resumen: El objetivo del presente trabajo es analizar las estrategias de búsqueda visual desarrolladas por un grupo de conductores expertos $(\mathrm{n}=5)$ y otro inexperto $(n=5)$ en una situación simulada de conducción en laboratorio. El registro de los movimientos oculares se hace a través del sistema de seguimiento de la mirada ASL SE5000 y se analizan el número, tiempo y localización de las fijaciones visuales. Los conductores observaron una secuencia de fotogramas específicos de conducción, para emitir seguidamente una respuesta verbal tras cada ensayo. Los resultados muestran que el grupo de conductores expertos e inexpertos elaboran diferentes estrategias de búsqueda visual. Estas diferencias se encuentran en un mayor tiempo de fijación del grupo de conductores experto cuando no se tiene en cuenta la localización de la fijación visual. También presenta un mayor número y tiempo de fijación en estímulos relevantes para la conducción (señales verticales y vehículos) con respecto al grupo inexperto. En cambio, este último grupo presenta un número y tiempo de fijaciones superior en localizaciones poco relevantes para la conducción. No existen diferencias entre grupos de conductores en la precisión de sus respuestas verbales, y tampoco una relación entre las variables perceptivas y la precisión de sus respuestas.
\end{abstract}

Palabras clave: Estrategias búsqueda visual; experiencia; conducción.

\section{Introducción}

En el ámbito de la seguridad vial, han sido numerosos los temas de estudio que han tratado de averiguar qué variables influyen en el rendimiento de la tarea de conducción y en la prevención de accidentes de tráfico. Algunas de las variables analizadas han sido la fatiga, el sueño durante la conducción, la exploración de las diferencias individuales entre sexos o edades de los conductores, la evaluación y análisis de los errores más frecuentes durante la conducción, la relación entre percepción errónea del riesgo y la sobreestimación de las propias habilidades con el riesgo de accidente, el efecto de la ingestión de distintas sustancias adictivas (alcohol y drogas) con la probabilidad de accidente, la educación vial, el entrenamiento de las habilidades para la conducción, y la importancia de los factores sociales en la conducción (Castro \& Martos, 1997).

La conducción de un vehículo es una actividad compleja, que incluye la realización coordinada de múltiples y diversas tareas en un entorno variable que, junto a la velocidad del desplazamiento y las continuas modificaciones estimulares en la situación de tráfico, exigen al conductor diversos cambios en su foco de visión. Por ello, aprender a conducir implica, entre otras habilidades, aprender a explorar visualmente el entorno en el que se conduce, con el objetivo de detectar e identificar con rapidez y precisión los eventos potencialmente relevantes (McKenna \& Crick, 1994).

\footnotetext{
* Dirección para correspondencia [Correspondence address]:

Juan Miguel Gómez-Valadés Horrillo. Avda. Universidad s/n, 10003,

Cáceres, Extremadura (España).Email: jmgomezvalades@gmail.com
}

Title: Visual search strategies in expert and novice drivers during the perception of driving scenes.

Abstract: The aim of this study is to analyze the visual search strategies of expert $(\mathrm{n}=5)$ and inexperienced drivers $(\mathrm{n}=5)$ during a simulated task of driving in laboratory. The ocular movements are recorded with an ASL SE5000 eye tracking system; and the number, time and location of these fixations are analyzed too. The drivers observed a sequence of specific frames of driving, and then they had to respond with a verbal respond after each trial. The results show that the expert and inexperienced group develop different visual search strategies. These differences are the higher time of fixation, independently of location fixation; and even the higher number and time of fixation on relevant stimuli during the driving (vertical signs and vehicles) in the expert group against to other group. Instead, the inexperienced group shows higher number and time on irrelevant stimuli during the driving. There are no differences between groups of drivers on the accuracy of their verbal response, and neither there is any relation between perceptual variables and the accuracy of their responses.

Key words: visual search strategies; experience; driving.
Una ineficaz percepción del entorno podría estar motivada por deficientes estrategias de búsqueda visual para explorar efectivamente los estímulos que aparecen en la carretera (Tejero, Pastor, \& Crespo, 2004), impidiendo a su vez una toma de decisiones acertada (McMorris, 1999) e, incluso, la anticipación ante posibles riesgos en la situación de conducción (Pradhan, Pollatsek, Knodler, \& Fisher, 2009). Todo ello podría convertirse en una de las causas que explicasen la mayor probabilidad que tienen los conductores inexpertos de estar implicados en situaciones de riesgo para la conducción (Chan, Pradhan, Pollatsek, Knodler, \& Fisher, 2010; Cooper, Pinili, \& Chen, 1995).

Tejero et al. (2004) realizaron una revisión de estudios sobre la exploración visual de conductores con distinta experiencia a partir del registro de los movimientos oculares, determinando que una de las variables que mejor discriminan a ambos colectivos de conductores es el tiempo de fijación visual, así como la mayor flexibilidad que poseen los conductores expertos en obtener información relevante para las situaciones cambiantes de conducción. Además, la duración de estas fijaciones oculares está considerada como un indicador de carga de procesamiento de la información que tienen los conductores, en función del nivel de complejidad que posea la situación estimular presentada (Konstantopoulos, Chapman, \& Crundall, 2010).

En la literatura, no existe uniformidad en los resultados encontrados, ya que las situaciones de tráfico presentadas en diferentes estudios mostraban diferentes demandas de procesamiento de información, por lo que no es factible realizar comparaciones respecto al análisis de las fijaciones visuales. De este modo, existen trabajos donde las fijaciones visuales 
son más breves en los conductores con menos experiencia que respecto a los expertos (Carter \& Laya, 1998; Dishart \& Land, 1998; Seya \& Nakayasu, 2008; Unema \& Röting, 1990); otros donde los conductores noveles realizaban fijaciones más largas (Chapman \& Underwood, 1998; Crundall, Underwood, \& Chapman, 1999; Konstantopoulos et al., 2010); y también algún estudio donde no se encontraban diferencias según la experiencia (Huestegge, Skottke, Anders, Müsseler, \& Debus, 2010).

De forma más específica, hay trabajos que postulan que no existen diferencias significativas en la duración media de las fijaciones de conductores noveles y conductores con más experiencia en un mismo tipo de entorno de tráfico, tanto en situación de investigación de conducción real (Crundall \& Underwood, 1998), como en situación de visualización de video-clips de tráfico (Crundall et al., 1999). Sin embargo, cuando se comparan las fijaciones en distintos tipos de carretera, la variable experiencia en la conducción sí que afecta a la duración media de las fijaciones, ya que aparecen diferencias significativas tanto en el grupo de conductores expertos, como en el grupo de conductores noveles, cuando se comparan diferentes tipos de carretera. De este modo, los conductores con mayor experiencia realizan fijaciones oculares más largas en carretera rural, ya que ésta plantea menor procesamiento estimular debido a su baja intensidad de tráfico. Sin embargo, la duración de sus fijaciones es menor en carretera urbana y de doble carril a fin de extraer información visual a partir de un número mayor de estímulos del entorno, realizando finalmente un mayor número de fijaciones visuales (Crundall y Underwood, 1998).

Además, las fijaciones visuales no suelen distribuirse espacialmente de forma proporcional, ya que hay zonas de la escena que reciben mayor número de fijaciones que otras, o de mayor duración respecto a otras zonas, considerándose éstas como fuentes de información relevantes. Recarte, $\mathrm{Nu}$ nes, López, y Recarte (1998) confirman que los eventos relevantes se producen principalmente sobre el eje horizontal de visión durante la conducción. Crundall et al. (1999) no encontraron diferencias en la exploración visual entre conductores expertos y principiantes, tanto en el eje horizontal como vertical, pero sí dentro del grupo experto, ya que varió su patrón de exploración visual en función de la vía de tráfico visualizada, comportamiento que no aparecía en el grupo de principiantes.

También se han realizado estudios que analizan los movimientos oculares en conductores expertos y noveles respecto a la localización de sus fijaciones visuales y la información relevante e irrelevante del entorno (Falkmer \& Gregersen, 2005; Konstantopoulos et al., 2010; Underwood, Chapman, Berger, \& Crundall, 2003; Underwood, Chapman, Bowden, \& Crundall, 2002); información para ajustar la trayectoria del vehículo (Dishart \& Land, 1998; Underwood, Chapman, Brocklehurst, Underwood, \& Crundall, 2003); e información que ayuda a anticipar un evento inesperado y peligroso (Chapman, Underwood, \& Roberts, 2002; Crundall et al., 1999; Hosking, Liu, \& Bayly, 2010; Huestegge et al., 2010; Underwood, Chapman, Berger et al., 2003). Destacar la aportación de Miltenburg y Kuiken (1990), quienes registraron los movimientos oculares de diferentes grupos de conductores mientras visualizaban simulaciones de vídeo de situaciones de conducción, concluyendo que los conductores con poca o media experiencia se fijaban antes en los objetos irrelevantes que el grupo de más experiencia; o la de Wikman, Nieminen, y Summala (1998), quienes verificaron que un mayor porcentaje de conductores inexpertos tienen un mayor tiempo de fijación sobre objetos distractores del interior del vehículo.

Algunas investigaciones en seguridad vial han desarrollado y aplicado diferentes simuladores de conducción, tratando de acercar lo máximo posible a los sujetos a la situación real de tráfico. Algunos ejemplos de ello son el simulador de conducción Sirca (Bayarri, Fernández, \& Pérez, 1993), o el SIMUVEG, un sistema avanzado de representación de escenas realistas dentro de un entorno invasivo, generado en el seno del grupo de investigación SINTEC (Simulación y Nuevas Tecnologías en Tráfico y Seguridad Vial) del INTRAS (Instituto Universitario de Investigación en Tráfico y Seguridad Vial) de la Universidad de Valencia. Velichkovsky, Rothert, Kopf, Dornhöfer, y Joos (2002) también utilizaron el simulador con acciones dinámicas para relacionar las fijaciones oculares y la capacidad de reacción ante la aparición de eventos peligrosos en conductores experimentados. Dichos autores concluyen que la duración de las fijaciones de estos sujetos aumentaba ante un inmediato peligro potencial, tal como ocurre en el trabajo de Chapman y Underwood (1998). En conducción real, Recarte y Nunes (2000) utilizaron un coche equipado con un sistema de presentación automática de estímulos visuales a los que los sujetos tenían que reaccionar mientras realizaban diferentes tipos de tareas cognitivas. Los resultados mostraron que la realización de tareas cognitivas durante la conducción produce un aumento del diámetro de la pupila, lo que indica un esfuerzo adicional en la conducción. Además, la realización de una tarea cognitiva provoca una disminución de la ventana visual, independientemente del tipo de vía, y sobre todo en tareas de procesamiento de imágenes visuales frente a tareas de procesamiento verbal. Posteriormente, los mismos Recarte y Nunes (2003) añaden que la actividad mental durante la conducción podría afectar a procesos tales como detección, identificación y selección de la respuesta.

Patten, Kircher, Östlund, Nilsson, y Svenson (2006), utilizando como tarea secundaria la detección por periferia de ciertos estímulos visuales, encontraron que los conductores con menos experiencia tenían tiempos de reacción mayores que los conductores con más experiencia. También, Summala (1998), Summala, Nieminen, y Punto (1996) demostraron que los conductores inexpertos tenían más dificultades que los expertos en seguir la trayectoria del vehículo cuando el estímulo a percibir estaba más alejado de la línea de fijación visual.

Hosking et al., (2010) concluyeron además que los motociclistas con más experiencia reaccionaban antes a los estí- 
mulos, y tenían un patrón de búsqueda visual más flexible que los conductores con menos experiencia. Similares resultados encontraron Huestegge et al. (2010), donde los conductores con más experiencia reaccionaban más rápido ante un evento peligroso. En este estudio se utilizaron como estímulos fotografías de escenas de conducción, presentadas durante dos segundos y con un segundo de separación entre cada una de ellas, ante las que había que reaccionar, si el sujeto lo consideraba, presionando un botón del teclado que había delante de él. Cada cinco fotografías, se realizaban preguntas al sujeto acerca de lo observado en las fotos. Las respuestas de los sujetos no fueron analizadas, pero servían de control para asegurar que los conductores estuvieran atentos a las fotografías.

El presente estudio trata de explorar y conocer, a través de un sistema de registro de los movimientos oculares, el proceso de búsqueda visual utilizado por conductores de diferente experiencia ante la visualización de escenas estáticas de tráfico, similares a las empleadas en pruebas escritas de evaluación de la aptitud para la conducción. Este estudio de carácter empírico, desde una perspectiva cognitiva de análisis del comportamiento humano, describe, analiza y compara las estrategias de búsqueda visual utilizadas por conductores expertos e inexpertos mediante simulación en laboratorio de diferentes situaciones de tráfico. Los participantes del estudio debían percibir, en un tiempo limitado, una serie de fotografías que representan situaciones específicas de tráfico, y responder acertadamente a una pregunta formulada por el equipo de investigación después de cada ensayo. Se espera que los conductores expertos desarrollen una estrategia de búsqueda visual distinta que la de los conductores inexpertos, caracterizada por orientar la visión hacia los estímulos relevantes, y con una mayor duración temporal de las fijaciones visuales sobre estos estímulos relevantes.

\section{Método}

\section{Participantes}

Para este trabajo, contamos con una muestra formada por 10 participantes, divididos en dos grupos: 5 conductores experimentados con una edad media de 29.20 años $(D T=7.75)$, una experiencia media de conducción de 10.40 años de permiso $(D T=7.70)$ y una media de $17800 \mathrm{~km}$ anuales $(D T=10.08)$; y 5 conductores noveles con una edad media de 19.80 años $(D T=1.40)$, una experiencia media de conducción de 37.20 días de permiso $(D T=26.00)$ y una media de 292 kilómetros anuales (DT=414.21). Todos los sujetos participaron voluntariamente en el estudio, previa firma de un informe de consentimiento de participación avalado por el comité de ética de la Universidad donde se llevó a cabo la investigación.

\section{Instrumentos}

Para el registro de las fijaciones visuales se utilizó el sistema tecnológico ASL Eye Tracking System SE5000 (Applied Sciences Laboratories $\left.{ }^{\mathrm{TM}}\right)$, que permite registrar en tiempo real el conjunto de fijaciones visuales realizadas por cada participante. Se trata de un sistema monocular que basa su funcionamiento en determinar, a partir de la superficie ocular, el diámetro de la pupila, su localización y el punto más brillante de la reflexión corneal. Con estos puntos, el software del sistema calcula constantemente el vector de visión en fóvea o visión central. Esto es posible gracias a una cámara de luz infrarroja que filma los movimientos del ojo izquierdo. Además, contiene en uno de sus laterales una pequeña cámara que filma la escena y un visor de cristal polarizado que refleja las imágenes de ambas cámaras. Dichas imágenes son grabadas e integradas por el software del sistema, dando como resultado una película que incluye el entorno percibido con el punto de fijación visual en fóvea superpuesto (Figura 1). El número, tiempo y localización de cada fijación visual conforma la estrategia de búsqueda visual utilizada por cada participante para percibir los estímulos relevantes en cada observación de situaciones de tráfico. El análisis del comportamiento visual se realizó a una frecuencia de 50 fotogramas por segundo, realizando un análisis fotograma a fotograma con un video Mini DV Sony DSR-30p.

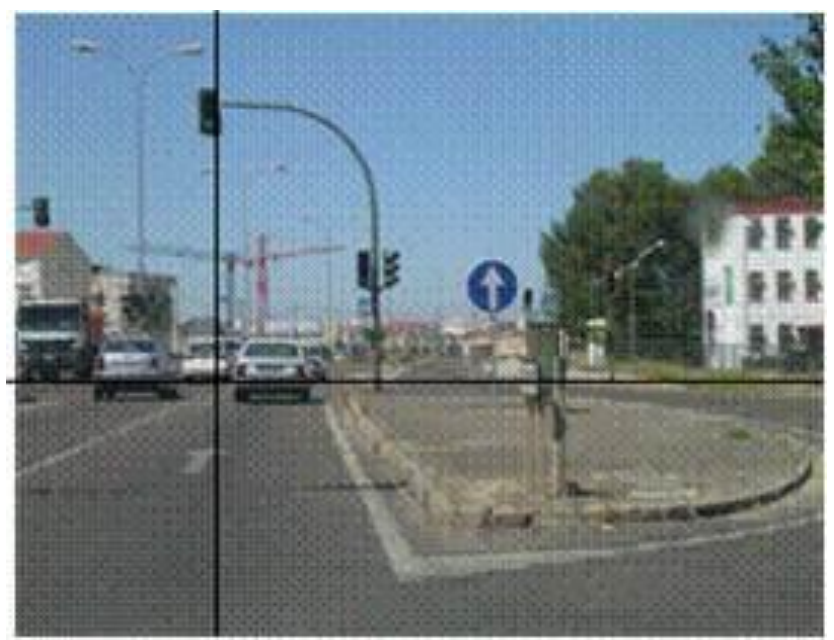

Figura 1. Ejemplo de fijación visual resultante en una escena de tráfico.

Se utilizó también un sistema automatizado de control de la información para presentar a todos los participantes la misma secuencia de ensayos con imágenes de tráfico. Así, la preparación y elaboración de dicha secuencia de ensayos se realizó a través del software Adobe ${ }^{\mathrm{TM}}$ Premiere $^{\mathrm{TM}}$ 5.1. Una vez editada y verificada dicha secuencia, se exportó la película para su posterior grabación y reproducción en un video digital. La captura y edición de los fotogramas de conducción se realizó en un computador equipado con una tarjeta de captura de video analógico y digital (Pinnacle ${ }^{\mathrm{TM}}$ DV500). La proyección de la secuencia se realizó mediante un video 
proyector sobre una pantalla de retroproyección de $5 \times 3 \mathrm{~m}$ (Figura 2).

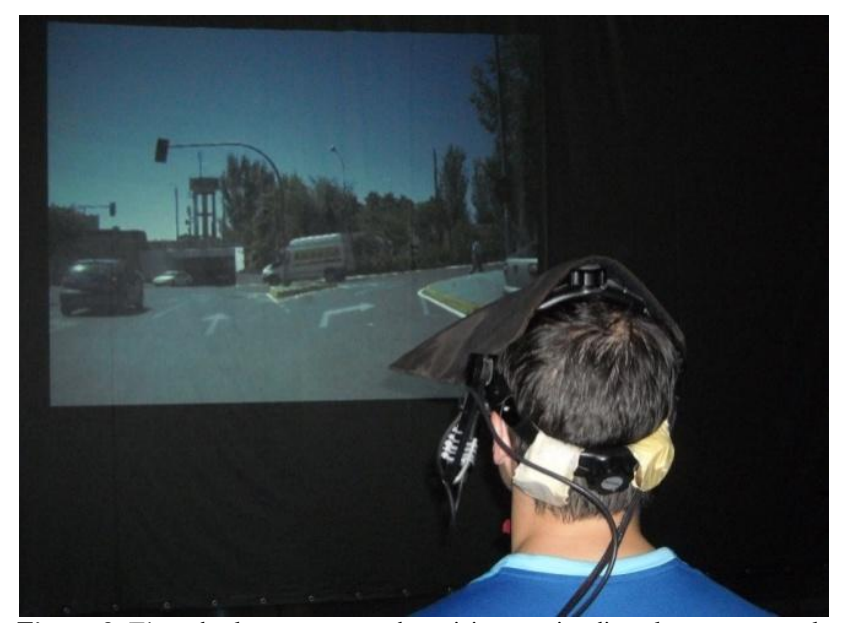

Figura 2. Ejemplo de ensayo con el participante visualizando una escena de tráfico

Analizamos la relación del factor experiencia en la conducción (2 niveles: experimentados y noveles), con las variables relacionadas con la percepción durante una situación de conducción, mediante el registro de las fijaciones visuales. En la presente investigación el concepto de fijación visual se entiende como el tiempo mínimo de $100 \mathrm{~ms}$ en el que la mirada se detiene en una misma localización espacial (Williams, Davids, \& Williams, 1999). En concreto, se distinguen el número de fijaciones visuales, o número de ocasiones en las que el participante mantiene el estímulo dentro de la visión en fóvea; el tiempo de fijación visual, o cantidad de tiempo (ms) que dedica el participante a mantener su visión en fóvea en dicho estímulo; y la localización de la fijación visual, entendida como la ubicación donde el participante fija su visión. La selección de nuestras localizaciones visuales se basó en el criterio utilizado por Tejero et al. (2004), tras su revisión de estudios relacionados con el análisis de movimientos oculares en distintas situaciones de tráfico. Se concretaron las siguientes localizaciones: señales verticales, semáforos, acera, calzadas, otros coches, marcas viales, peatones y otros estímulos irrelevantes (p.e. árboles, carteles de publicidad, construcciones, etc.).

También se analiza la precisión de las respuestas verbales, entendida como la cantidad de aciertos emitidos por el participante en función de su respuesta dada a la cuestión planteada en cada situación de tráfico. Se solicita una respuesta verbal y no motora para evitar que la exploración visual pudiera estar influencia por el grado de adquisición alcanzado en otras habilidades más básicas como el grado de control de desplazamiento del vehículo (Chapman \& Underwood, 1998). Este procedimiento de acceso a la información ya fue utilizado anteriormente por Underwood, Chapman, Berger et al. (2003), que tenía como objetivo conocer la relación entre la experiencia de conducción, la atención y el recuerdo de información. Consistía en percibir secuencias de video con diferentes escenas de tráfico, que se detenían y ocluían en determinados momentos temporales. En esos momentos, se le preguntaba al participante acerca de lo observado en la secuencia de video, teniendo que recordar ciertos eventos anteriormente visualizados. Las respuestas correctas se relacionaban con los datos de las fijaciones visuales.

\section{Procedimiento}

Cada participante, sentado frente a la pantalla a una distancia de 3 metros, visualizó 17 situaciones distintas y específicas de tráfico, de las cuales las 2 primeras son de habituación del participante al protocolo de medida. Estas situaciones fueron presentadas a través de fotografías, similares a las utilizadas en los test de conducción, y representativas de dichos escenarios de tráfico. Dichas fotografías, de un tamaño similar a un escenario real de conducción $(2.05 \mathrm{~m}$ de ancho x $1.60 \mathrm{~m}$ de alto), proyectaban un ángulo visual de $39.2^{\circ}$ horizontal y $30.6^{\circ}$ vertical.

Cada ensayo consistía en la presentación durante 3 segundos del fotograma correspondiente, tiempo durante el cual el participante debía percibir los estímulos relevantes para una conducción adaptada a dicha situación específica. Una vez acabado dicho tiempo, y antes de la presentación del siguiente ensayo, se requería a dicho participante responder de forma dicotómica (verdadero o falso) a una pregunta en relación con lo percibido en dicha imagen (p.e. "ipuedes adelantar en el siguiente tramo de carretera?”, “ipuedes girar a la izquierda o la derecha en el siguiente cruce?”.). Entre cada ensayo, el sujeto debía mantener la visión en un recuadro situado en el centro de la proyección, para que todos los sujetos empezaran la visualización desde una misma referencia espacial. Este proceso de visualización de situaciones y respuesta verbal se repitió tras cada ensayo hasta completar la secuencia. Las respuestas verbales de los participantes fueron anotadas por el personal de investigación en una plantilla de registro creada para tal efecto.

\section{Análisis de datos}

Se realizaron las pruebas de normalidad (KolmogorovSmirnov y Shapiro-Wilks) y de homogeneidad de varianza de Levene a todas las variables dependientes de estudio, decidiendo aplicar la prueba no paramétrica de Mann-Whitney para 2 muestras independientes en aquellas variables en que no exista una distribución normal de datos. Para las variables que cumplieron dicho supuesto de normalidad se aplicó la prueba $t$ de Student de muestras independientes. Además, estos análisis se realizan en función de la localización de la fijación visual e independientemente de dicha localización, para determinar si existen diferencias en el número y tiempo de fijación visual en función de la experiencia en la conducción. También se realiza un análisis correlacional para conocer si las variables perceptivas están relacionadas con la precisión de las respuestas verbales emitidas por los grupos de con- 
ductores, e indagar si la estrategia de búsqueda visual elaborada permite a dichos conductores responder efectivamente a una cuestión relativa a la secuencia de conducción presentada. Para la realización de todas estas pruebas estadísticas se empleó el paquete estadístico SPSS 17.0.

\section{Resultados}

En primer lugar se presentan los resultados descriptivos de cada grupo de conductores en cada una de las localizaciones de la fijación visual (Tabla 1). Las localizaciones más relevantes para ambos grupos, tanto en número como en tiempo de fijación visual, son las de señal vertical y otros coches. En cambio, las localizaciones con menor número y tiempo de fijaciones visuales son la acera, semáforos y peatones.

La prueba $U$ de Mann-Whitney para dos muestras independientes, teniendo en cuenta la localización de la fijación visual, muestra que existen diferencias entre el grupo de conductores expertos y novel para las localizaciones señales verticales, otros coches y otros estímulos. En concreto, se obtienen diferencias significativas entre grupos para dichas localizaciones, tanto en el número como en el tiempo de las fijaciones visuales. Los sujetos con más experiencia tienen más fijaciones y más largas que los noveles a estímulos relevantes para la situación de conducción: número de fijaciones en señales verticales $(U=1966.5 ; p<.05)$, tiempo de fijación en señales verticales $(U=2127.5 ; p<.05)$, número de fijaciones en otros coches $(U=2201 ; p<.05)$ y tiempo de fijación en otros coches $(U=2242 ; p<.05)$. También, dicho grupo muestra menos fijaciones visuales y de menor duración sobre estímulos irrelevantes: número de fijaciones en otros estímulos $(U=1639 ; p<.001)$ y tiempo de fijación a otros estímulos $(U=1714.5 ; p<.001)$. Además, la prueba de Levene confirma que no existe homogeneidad de varianza entre grupos de conductores tanto a nivel de número $(F=11.08 ; p<.05)$ como de tiempo de fijaciones $(F=6.09 ; p<.05)$.

Tabla 1. Estadísticos descriptivos $(M \pm D T)$ de cada grupo respecto al número fijaciones (NF) y tiempo de fijaciones (TF) en cada localización de la fijación visual.

\begin{tabular}{|c|c|c|c|c|}
\hline & \multicolumn{2}{|c|}{ Grupo Experto } & \multicolumn{2}{|c|}{ Grupo Novel } \\
\hline & $\mathrm{NF}$ & TF & $\mathrm{NF}$ & TF \\
\hline Señal vertical & $3.07 \pm 1.98$ & $1201.87 \pm 810.12$ & $2.08 \pm 1.87$ & $896.76 \pm 861.74$ \\
\hline Semáforo & $.51 \pm .97$ & $168.53 \pm 352.75$ & $.28 \pm .69$ & $90.81 \pm 243.49$ \\
\hline Acera & $.15 \pm .42$ & $33.87 \pm 102.87$ & $.09 \pm .29$ & $26.22 \pm 91.25$ \\
\hline Calzada & $.87 \pm 1.50$ & $184.00 \pm 325.32$ & $1.24 \pm 1.65$ & $265.41 \pm 387.08$ \\
\hline Otros coches & $1.81 \pm 1.85$ & $486.93 \pm 541.16$ & $1.26 \pm 1.70$ & $340.54 \pm 473.74$ \\
\hline Marca vial & $1.48 \pm 1.96$ & $338.13 \pm 432.75$ & $1.26 \pm 1.60$ & $264.86 \pm 342.25$ \\
\hline Peatones & $.55 \pm 1.24$ & $153.33 \pm 365.19$ & $.35 \pm .89$ & $120.54 \pm 345.65$ \\
\hline Otros estímulos & $1.29 \pm 1.40$ & $324.27 \pm 386.80$ & $2.68 \pm 2.27$ & $637.30 \pm 575.12$ \\
\hline
\end{tabular}

Cuando se realiza de nuevo la prueba $U$ de MannWhitney, sin tener en cuenta ahora la localización de las fijaciones visuales $(U=233533 ; p<.05)$, el grupo experto de conductores presenta un mayor tiempo de fijación visual (297.45 ms) con respecto al grupo de conductores noveles (286.90 ms). Para la variable número de fijaciones visuales, también independientemente de la localización de la fijación, se realiza una prueba $t$ de muestras independientes, encontrando que no existen diferencias significativas entre grupos en el promedio de número de fijaciones visuales.

También se realiza una prueba $t$ para muestras independientes para verificar si existen diferencias en el número de respuestas verbales acertadas según la experiencia de los conductores, encontrándose que no existen diferencias en la media de respuestas correctas realizadas entre grupos de conductores (expertos: $M=9.80$ aciertos, $D T=1.64$; noveles: $M=8.20$ aciertos; $D T=1.92)$. Para corroborar estos resultados, se realizó además la prueba no paramétrica $U$ de Mann-Whitney, ya que trabajamos con una muestra pequeña, y tampoco se encontraron diferencias entre los dos grupos de conductores.

Respecto a la relación que tiene el comportamiento visual y la precisión de las respuestas, el análisis correlacional entre las variables perceptivas y la cantidad de respuestas acertadas, según localización de la fijación visual, muestra que el grupo experto obtiene un mayor número de aciertos cuanto menor número y tiempo de fijaciones dedican a la percepción de los estímulos acera y peatones. En concreto, los estadísticos son para la variable NF_acera $(r=-.344$; $p<.01)$, variable TF_acera $(r=-.280 ; \quad p<.05)$, variable NF_peatones $(r=-.358 ; p<.01)$ y variable TF_peatones $(r=-$ $.390 ; p<.01)$.

El análisis de aquellos ensayos en los que los participantes fijan visualmente el estímulo relevante de cada situación de conducción, muestra que los conductores con mayor experiencia consiguen un mayor porcentaje de respuestas acertadas $(67 \%)$ que los sujetos con menor experiencia (57\%), aunque no de manera significativa $(U=1984.5 ; p=.236)$. Al realizar un análisis correlacional entre el comportamiento visual y los aciertos en las respuestas verbales emitidas, sólo en aquellos ensayos en los que los participantes fijan previamente sobre el estímulo relevante, se observa de nuevo que el grupo experto consigue correlaciones negativas en las mismas y anteriores localizaciones: NF_acera $(r=-.410$; $p<.001)$, TF_acera $(r=-.362 ; p<.01), \mathrm{NF} \_$peatones $(r=-.396$; $p<.01)$ y TF_peatones $(r=-.429 ; p<.01)$. 


\section{Discusión}

Los resultados muestran que el grupo de conductores expertos tiene una estrategia de búsqueda visual diferenciada respecto al grupo de conductores inexpertos durante las situaciones de tráfico observadas. Esta diferenciada estrategia de exploración visual seguida por los conductores según su nivel de experiencia coincide con las aportaciones de Tejero et al. (2004), quienes afirman en su trabajo de revisión que las fijaciones no se distribuyen de forma proporcional, ni en cantidad ni en tiempo, por la escena de conducción.

En nuestro estudio, el grupo con mayor experiencia en conducción ha obtenido un mayor número de fijaciones en localizaciones tales como señales verticales y otros coches. Estas localizaciones pueden ser relevantes para el rendimiento en la tarea de conducción, ya que dichos estímulos podrían proporcionar información significativa y suficiente a los conductores para guardar la separación y distancia de seguridad respecto a otros vehículos (en el caso de la localización de otros coches), o proporcionar información precisa y adaptada para tomar decisiones relativas a qué hacer ante señales anunciadoras de peligro en la carretera, de reglamentación y prioridad, o incluso ante señales de indicación del sentido de la marcha (en el caso de la localización de señales verticales). Esta diferencia en la cantidad de fijaciones según la localización espacial y experiencia en la conducción ha sido anteriormente verificada en trabajos como los de Unema y Röting (1990), Carter y Laya (1998), Dishart y Land (1998), o Seya y Nakayasu (2008).

Además, los conductores noveles han fijado más su visión, tanto en número como en tiempo, en otros estímulos irrelevantes para la conducción tales como carteles de publicidad u objetos del interior del vehículo. Dicho resultado coincide con las aportaciones de Miltenburg y Kuiken (1990), quienes afirman, en su estudio con conductores de diferente experiencia, que los grupos con poca y media experiencia fijan antes su visión en los estímulos irrelevantes en comparación al grupo de mayor experiencia en la conducción.

En el análisis correlacional, el grupo experto obtiene una relación negativa entre la cantidad de aciertos y el número y tiempo de fijación dedicada a otras localizaciones tales como la acera y los peatones, ya sea para el total de situaciones de conducción percibidas o sólo en aquellas donde los conductores fijaron visualmente en el estímulo relevante. De esta manera, cuanto más veces y tiempo atienda a dichas localizaciones no relevantes va a responder de forma más imprecisa a la pregunta formulada por el investigador en relación a la situación específica de tráfico visualizada con anterioridad. Este comportamiento creemos que es debido a que dichas localizaciones no tienen que ver directamente con la propia situación de tráfico y sí con la información complementaria presente en el contexto estimular, pero que apenas ayuda a mejorar la eficacia en la tarea. Por este motivo, dicho grupo reorienta su visión hacia otro tipo de estímulos relevantes para la situación de tráfico, tales como las señales verticales y otros coches, que ayudaría a los conductores a tomar decisiones acertadas en función de cada situación presentada.

Este comportamiento visual adaptado del grupo experto responde a estrategias perceptivas subyacentes del participante, con un carácter no aleatorio (Tejero et al., 2004), y mediatizadas por el conocimiento y experiencia que se tiene de la tarea (Williams et al., 1999). Dicho comportamiento visual de búsqueda de estímulos relevantes para la conducción es posible gracias a la elaboración de patrones visuales más flexibles (Crundall et al., 1999; Hosking et al., 2010). Sin embargo, este grupo de conductores expertos, a pesar de mostrar una estrategia de búsqueda visual distinta al grupo inexperto, no consigue mayor cantidad de aciertos en sus respuestas verbales. Este comportamiento refuerza las conclusiones de Underwood, Chapman, Berger et al. (2003), quienes encontraron que los conductores expertos y noveles tuvieron un recuerdo similar, sin diferencias significativas, de eventos relevantes en distintas secuencias de tráfico, no siendo así ante los eventos irrelevantes, donde los expertos recordaron un mayor número de ellos. Ello contradice la aportación de McMorris (1999), quien sugiere que una percepción eficaz del entorno podría ayudar a los sujetos a tomar decisiones; o la de Chan et al. (2010), quienes afirman que una ineficaz estrategia de búsqueda visual podría ser una de las causas que predisponen a los conductores inexpertos, junto a su falta de experiencia y capacidad de anticipar los posibles riesgos (Pradhan et al., 2009), a generar situaciones de riesgo o accidente (Cooper et al., 1995).

Posiblemente, el tipo de respuesta solicitada, una respuesta verbal y no motora, impida al grupo de conductores expertos utilizar esa información ventajosa captada a través de su canal visual, por tratarse de una respuesta poco cercana o adaptada a la situación real de conducir. De hecho, en trabajos donde se solicitó una respuesta motriz (Hosking et al., 2010; Patten et al., 2006) sí se ha demostrado que el grupo de expertos reaccionaba antes, incluso también con estímulos presentados en la periferia del campo de visión. También el tipo de imagen proyectada, estática, con ausencia de información direccional, temporal y secuencial podría limitar y condicionar el uso avanzado de estímulos relevantes para la toma de decisiones y la respuesta en los conductores con más experiencia.

\section{Conclusiones}

La principal aportación del trabajo ha sido realizar, desde una perspectiva cognitiva, una descripción del comportamiento visual de conductores de diferente experiencia, en situación controlada de laboratorio mediante la observación de diferentes situaciones de tráfico, a través de un procedimiento automatizado de registro de los movimientos oculares. Además, la utilidad de este estudio es la aplicación de un protocolo de registro en tiempo real de las estrategias de búsqueda visual en tareas de observación de situaciones de tráfico, mediante la proyección de diferentes escenas de tráfico. 
La experiencia en la conducción fomenta el desarrollo de un patrón de búsqueda visual diferenciado en el que el grupo experto de conductores presenta un mayor número y tiempo de fijaciones visuales orientada hacia estímulos relevantes, tales como las señales verticales y otros coches. Sin embargo, al tener dos grupos de edad y experiencia muy diferentes existe la dificultad en separar los efectos de la edad de los efectos de la experiencia de conducción, lo que impide conocer la influencia real de cada variable por separado en la elaboración del patrón visual.

En futuras investigaciones también sería interesante conocer si simulando las condiciones reales de conducción a través de proyecciones dinámicas o solicitando respuestas

\section{Referencias}

Bayarri, S., Fernández, M., \& Pérez, M. (1993). El simulador de conducción Sirca: Una herramienta de realidad virtual para la investigación y la formación en seguridad vial. Acta. Aplicaciones de Computación y Telemática Avanzada, 1 (1), 64-69.

Carter, C. J., \& Laya, O. (1998). Drivers' visual search in a field situation and in a driving simulator. En A. G. Gale, I. D. Brown, C. M. Haslegrave, S. P. Taylor (Eds.), Vision in vehicles - VI (pp. 21-31). Oxford: Elsevier

Castro, C., \& Martos, F. J. (1997). Aportaciones de la psicología a la seguridad vial. Una revisión de las áreas más importantes. Boletín de Psicología, 54, 25-52.

Chan, E., Pradhan, A. K., Pollatsek, A., Knodler, M. A., \& Fisher, D. L. (2010). Are driving simulators effective tools for evaluating novice drivers' hazard anticipation, speed management, and attention maintenance skills? Transportation Research Part F, 13 (5) 343-353.

Chapman, P. R., \& Underwood, G. (1998). Visual search of driving situations: danger and experience. Perception, 27, 951-964.

Chapman, P. R., Underwood, G., \& Roberts, K. (2002). Visual search patterns in trained and untrained novice drivers. Transportation Research Part F, 5 (2), 157-167.

Cooper, P. J., Pinili, M., \& Chen, W. (1995). An examination of the crash involvement rates of novice drivers aged from 16 to 55. Accident Analysis and Prevention, 27 (1), 89-104

Crundall, D., \& Underwood, G. (1998). Effects of experience and processing demands on visual information acquisition in drivers. Ergonomics, 41 (4), 448-458.

Crundall, D. E., Underwood, G., \& Chapman, P. R. (1999). Driving experience and the functional field of view. Perception, 28, 1075-1087.

Dishart, D. C., \& Land, M. F. (1998). The development of the eye movement strategies of learner drivers. En G. Underwood (Ed.), Eye guidance in reading and scene perception (pp. 419-429). Oxford: Elsevier.

Falkmer, T., \& Gregersen, N. P. (2005). A comparison of eye movement behavior of inexperienced and experienced drivers in real traffic environments. Optometry and vision science, 82 (8), 732-739.

Hosking, S. G., Liu, C. C., \& Bayly, M (2010). The visual patterns and hazard responses of experienced and inexperienced motorcycle riders. Accident analysis and prevention, 42 (1), 196-202

Huestegge, L., Skottke, E. M., Anders, S., Müsseler, J., \& Debus, G. (2010). The development of hazard perception: dissociation of visual orientation and hazard processing. Transportation Research Part F, 13 (1), 1-8.

Konstantopoulos, P., Chapman, P., \& Crundall, D. (2010). Driver's visual attention as a function of driving experienc and visibility. Using a driving simulator to explore drivers' eye movements in day, night and rain driving. Accident analysis and prevention, 42 (3), 827-834.

McMorris, T. (1999). Cognitive development and the acquisition of decision-making skills. International Journal of Sport Psychology, 30 (2), 151-172.

McKenna, F. P., \& Crick, J. (1994). Hazard perception in drivers: a methodology for testing and training. Contractor Report 313. Transport Research Laboratory, Crowthorne. motrices a los conductores (e.g. mover volante, pisar freno, meter una marcha) seguirían existiendo diferencias entre conductores expertos e inexpertos a nivel de comportamiento visual pero también de respuesta. En el futuro, sería de utilidad definir patrones de búsqueda visual de referencia, con un número mayor de conductores, y en situaciones más específicas de conducción o de distinta complejidad estimular (diferencias en las estrategias visuales según el tipo de vía: carreteras urbanas, ineterurbanas, rurales,...), que permitiesen a los conductores inexpertos mejorar su capacidad de reacción y respuesta ante los estímulos presentes en las mismas.

Miltenburg, P. G. M., \& Kuiken, M. J. (1990). The effect of driving experience on visual search strategies: Results of a laboratory experiment. Haren, Groningen: Rijksuniversiteit Groningen.

Patten, C. J. D., Kircher, A., Östlund, J., Nilsson, L., \& Svenson, O. (2006). Driver experience and cognitive workload in different traffic environments. Accident analysis and prevention, 38 (5), 887-894.

Pradhan, A. K., Pollatsek, A., Knodler, M., \& Fisher, D. L. (2009). Can younger drivers be trained to scan for information which will reduce their risk in roadway traffic scenarios that are hard to identify as hazardous? Ergonomics, 52 (6), 657-673.

Recarte, M. A., \& Nunes, L. M. (2000). Effects of verbal and spatial-imagery tasks on eye fixations while driving. Journal of Experimental Psychology: Applied. 6 (1), 31-43.

Recarte, M. A., \& Nunes, L. M. (2003). Mental workload while driving: effects on visual search, discrimination, and decision making. Journal of experimental psychology: Applied, 9 (2), 119-137.

Recarte, M. A., Nunes, L. M., López, R., y Recarte, S. (1998). Recursos atencionales y parámetros oculares en la conducción. En J. Botella y V. Ponsoda (Eds.), La atención: un enfoque pluridisciplinar (pp. 373-385). Valencia: Promolibro.

Seya, Y., \& Nakayasu, H. (2008). Visual search of trained and untrained drivers in a driving simulator. Japanese Psychological Research, 50 (4), 242252.

Summala, H. (1998). Forced peripheral vision driving paradigm: evidence for the hypothesis that car drivers learn to keep in lane with peripheral vision. En A.G. Gale, I. D. Brown, C. M. Haslegrave, S.P. Taylor (Eds.), Vision in vehicles - VI (pp. 51-60). Oxford: Elsevier.

Summala, H. Nieminen, T., \& Punto, M. (1996). Maintaining lane position with peripheral visión during in-vehicle tasks. Human Factors, 38 (3), 442-451.

Tejero, P., Pastor, G., \& Crespo, A. (2004) Exploración visual y movimientos oculares en conductores con distinta experiencia. Una revisión. Anales de psicología, 20 (1), 127-145.

Underwood, G., Chapman, P., Berger, B., \& Crundall, D. (2003). Driving experience, attentional focusing, and the recall of recently inspected events. Transportation Research Part F, 6 (4), 289-304.

Underwood, G., Chapman, P., Bowden, K., \& Crundall, D. (2002). Visual search while driving: skill and awareness during inspection of the scene. Transportation Research Part F, 5 (2), 87-97.

Underwood, G., Chapman, P. Brocklehurst, N., Underwood, J., \& Crundall, D. (2003). Visual attention while driving: sequences of eye fixations made by experienced and novice drivers. Ergonomics, 46 (6), 629-646.

Unema, P., \& Rötting, M. (1990). Differences in eye movements and mental workload between experienced and inexperienced motor-vehicle drivers. En D. Brogan (Ed.), Visual search. London: Taylor \& Francis.

Velichkovsky, B. M., Rothert, A., Kopf, M., Dornhöfer, S. M. \& Joos, M. (2002). Towards an express-diagnostics for level of processing and hazard perception. Transportation Research Part F, 5 (2), 145-156. 
Wikman, A. S., Nieminen, T., \& Summala, H. (1998). Driving experience and time-sharing durin in-car tasks on roads of different width. Ergonomics, 41(3), 358-372.
Williams, A. M., Davids, K., \& Williams, J. G. (1999). Visual perception and action in sport. London: E \& FN Spon.

(Artículo recibido: 04-07-2011, revisión: 01-12-2011, aceptado: 14-12-2011) 